\title{
53. Jahrestagung
}

der Deutschen Gesellschaft für Unfallheilkunde e.V.

22.-25. November 1989, Berlin

Kongreßthemen: Schock - Kniegelenksnahe Frakturen - Indikationsstellung bei Sportverletzungen - Komplexe Handverletzungen - Osteosynthese kindlicher Schaftfrakturen - Experimentelle Unfallchirurgie Freie Themen - Kuratorium ZNS - EDV-Dokumentation - Aktuelle Stunde - Begutachtung - Diskussionsrunde - Wissenschaftliches Filmprogramm - Wissenschaftliche Ausstellung - Schlußveranstaltung

Präsident: K.P. Schmit-Neuerburg Redigiert von: J. Probst

Springer-Verlag Berlin Heidelberg New York London Paris Tokyo Hong Kong Barcelona 


\section{Inhaltsverzeichnis}

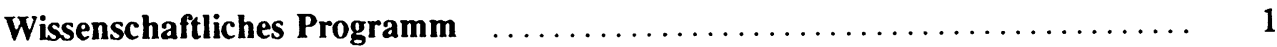

Eröffnungssitzung $\ldots \ldots \ldots \ldots \ldots \ldots \ldots \ldots \ldots \ldots \ldots \ldots \ldots \ldots \ldots \ldots \ldots \ldots \ldots \ldots \ldots$

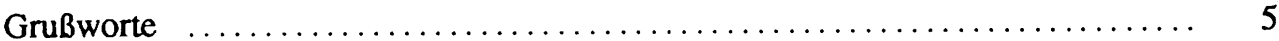

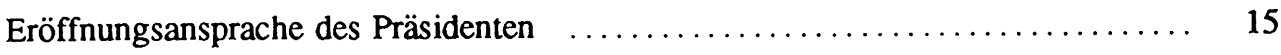

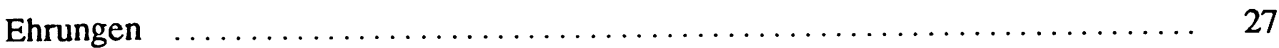

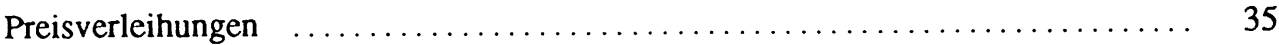

Festvortrag

Risiko und Verantwortung (F. Böckle) $\ldots \ldots \ldots \ldots \ldots \ldots \ldots \ldots \ldots \ldots \ldots \ldots \ldots \ldots \ldots \ldots \ldots$

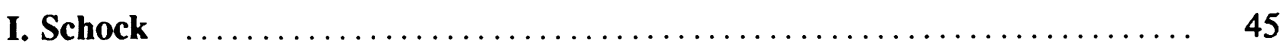

Pathophysiologie, Grundlagenforschung $\ldots \ldots \ldots \ldots \ldots \ldots \ldots \ldots \ldots \ldots \ldots \ldots$

Startreaktionen des traumatischen Schocks - Zelluläre Reaktionen

(Th. Joka)

Startreaktionen des posttraumatischen Schocks - Reperfusionsschäden

(G. Schlag und H.Redl)

Immunologische Konsequenzen des Trauma. Cytokine als Mediatoren der traumainduzierten immunologischen Defekte (E. Faist, M. Storck und W. Ertel) $\ldots 60$

Diagnostik - Präklinische Auswirkungen der pathologischen Erkenntnisse ...... 71

Wertigkeit von Score-Systemen (H.-J. Oestern und K. Kabus) $\ldots \ldots \ldots \ldots \ldots \ldots$

Klinische Diagnostik des Schocks (L. Schweiberer und D. Nast-Kolb) . . . . . . 79

Posttraumatische Frühveränderungen des humoral-granulocytären Abwehrsystems und der pulmonalen capillär-alveolären Permeabilität (A. Dwenger, G. Regel, Th. Joka und J.A. Sturm)

Mediatoren des traumatisch-hämorrhagischen Schockgeschehens mit neuer klinischer Relevanz (D. Nast-Kolb, Ch. Waydhas, M. Jochum, K.-H. Duswald und L. Schweiberer) 
Der Einfluß des Operationstraumas auf die Verletzungsantwort des Organismus beim polytraumatisierten Patienten (F. Bonnaire, U.Schöffel und E. H. Kuner) ...

Therapeutische Auswirkungen der pathophysiologischen Erkenntnisse - Frühphase

Wertigkeit der Frühbeatmung beim posttraumatischen Schock

(Th. Prien, J. Meyer und P. Lawin)

Zeitpunkt und Wertigkeit der operativ-neurochirurgischen Versorgung

beim Schockpatienten (W. Grote und R. Kalff)

Spezielle Aspekte des Schocks

Die Bedeutung des Schocks für die Verfahrenswahl bei Oberschenkelfrakturen

(K. Wenda, G. Ritter, J. Ahlers und J. Degreif)

Welchen Stellenwert hat die Analyse von Blutgasen in der Therapie

Schwerverletzter (A. Seekamp, G. Regel, J.A. Sturm und H. Tscherne)

Gängige Infusionsschemata und tatsächlicher Substitutionsbedarf in der

Akutphase bei Schwerverbrannten (F. Weyer, A. Grabosch, R. Büttenmeyer

und J.C. Bruck)

Die Bedeutung des hämorrhagisch-traumatischen Schocks und der

Thoraxverletzung für die Prognose nach Polytrauma

(Ch. Waydhas, D. Nast-Kolb, A. Trupka, M. Jochum und L. Schweiberer)

Ist das akute Nierenversagen nach Polytrauma therapeutisch zu beeinflussen?

(H.-C.Pape, G. Regel, J.A. Sturm und H. Tscheme) $\ldots \ldots \ldots \ldots \ldots \ldots \ldots \ldots$

Diskussion: Spezielle Aspekte des Schocks

Therapie in der Spätphase

Operative unfallchirurgische Versorgung nach Schock in der Spätphase -

Thorax und Abdomen (N. Rohm)

Operative unfallchirurgische Versorgung nach Schock -

Becken und Extremitäten (W. Fleischmann und L. Kinzl)

Surfactant-Substitution bei ARDS (Th. Joka, U. Obertacke, M. Reuter

und K.-P. Schmit-Neuerburg)

Posttraumatischer Anstieg der Leber- und Pankreasenzyme.

Trauma- oder Schockfolge? (M. Kahle, J. Lippert und R.D. Filler)

Aggressive Volumentherapie zur Prävention des akuten posttraumatischen

Nierenversagens (U. Finke, D. Neveling, Ch. Josten und G. Muhr) 
Leberverletzungen beim polytraumatisierten Patienten

(J. Erhard, H.J. Coone und U. Obertacke) $\ldots \ldots \ldots \ldots \ldots \ldots \ldots \ldots \ldots \ldots \ldots$

Trauma, Schock, Darmparalyse, Sepsis - eine Kausalität?

(J. Brand, D. Neveling, A. Ekkernkamp und G. Muhr) $\ldots \ldots \ldots \ldots \ldots \ldots \ldots \ldots \ldots$

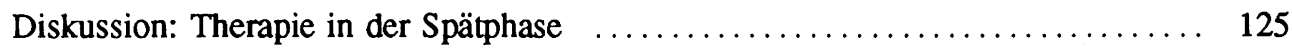

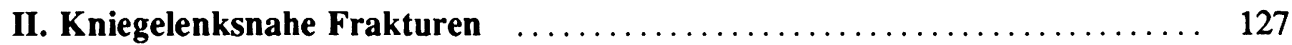

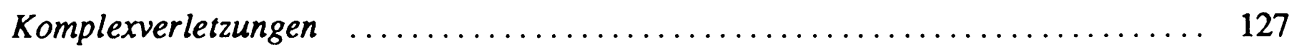

Diagnose und Erstbehandlung (U.Pfister und U. Harmel) $\ldots \ldots \ldots \ldots \ldots \ldots \ldots 127$

Definitive Versorgung von Komplexverletzungen des Kniegelenkes

(W. Mutschler und C. Burri)

Das komplexe Trauma des Kniegelenkes

(H. Reilmann, M. Blauth und P. Lobenhoffer)

Therapeutisches Konzept bei ipsilateralen kniegelenksnahen Frakturen von Femur und Tibia (J.Freistühler, P.-M. Hax und G. Hierholzer)

Die kniegelenksüberbrückende Fixateur-externe-Osteosynthese als therapeutisches Konzept bei Problemfrakturen

(M. Roesgen, M.Zander, M. Körber und G. Hierholzer)

Primär endoprothetisches Vorgehen bei kniegelenksbeteiligenden Frakturen ein neues Konzept zur frühen Rehabilitation vorwiegend älterer Patienten

(A. Benthien, C. Lütten und W. Thomas)

Patella

Biomechanik, Operationstechnik und Ergebnisse der Patellafrakturen

(U.Holz, F.W. Thielemann und B.Zahedi)

Indikation, Technik und Ergebnisse der konservativen und operativen

Behandlung bei 126 Patellafrakturen (M. Schax, R. Letsch, H. Gruthölter

und K.-P. Schmit-Neuerburg)

Die Patellektomie - unsere Erfahrungen und Ergebnisse

(G. Herold, M. Hess und V. Kohler)

Die Versorgung der Patellafraktur - eine Operation für den Anfänger?

(Ch. Jürgens, H.-R. Kortmann, J. Schultz und Ch. Eggers)

Zur konservativen Therapie der Patellafraktur

(R. Jaskulka, A. Chrysopoulos und G. Ittner)

Diskussion: Patella 
Tibiakopffrakturen - Diagnostik, Klassifikation und Therapie

(H. Tscherne und P. Lobenhoffer)

Indikation, Technik und Ergebnisse bei konservativer Therapie

der Tibiakopffrakturen (R. Szyszkowitz und P. Hofer)

Vergleichende Darstellung von 236 operativ und konservativ

behandelten Tibiakopffrakturen - Indikation, Technik und Ergebnisse

(P. Münst, W.Schlickewei, C. Krämer und E.H. Kuner)

Bericht über 212 operierte Schienbeinkopffrakturen

(R. Plaue, A. Hummel und O. Jandewerth)

Vorgehensweise bei zusätzlicher Meniscusverletzung nach Tibiakopffraktur

(L. Rudig, J. Ahlers und M. Lengsfeld)

Die externe Stabilisierung kniegelenksnaher Unterschenkelfrakturen -

Eine alternative Behandlungsmethode (R. Schlenzka, L. Gotzen und A. Boczek) . . 174

Die operative Behandlung von kniegelenksnahen Tibiatrümmerfrakturen

mit Fixateur externe und Minimal-Schraubenosteosynthesen

(G. Oedekoven, B. Claudi und B.Stübinger)

Therapiekonzept beim proximalen Unterschenkelbruch: Transfixation und frühzeitiger Verfahrenswechsel (W. Knopp, H.J. Menne, G. Muhr und K. Neumann)

Die Versorgung von Tibiakopffrakturen mit der Gabelplatte nach Streli.

Ergebnisse einer Nachuntersuchung von 94 Frakturen bei 92 Patienten

(E. Foltin, M. Fischmeister und W. Wurdinger)

Diskussion: Tibia

Femur

Therapeutisches Konzept bei der distalen Femurfraktur mit Gelenkbeteiligung

(N. Haas, P.Schandelmaier und C. Krettek)

Spätergebnisse distaler Femurfrakturen - AO-Dokumentation

(P. Matter, R. Berbug und M. Bühler)

Distale Femurfraktur des Erwachsenen - Überprüfung des Therapiekonzeptes durch Nachuntersuchung 1980 bis 1987 (A. Imdahl, G. Siebler, O. Ocker und E.H. Kuner)

Alignement und Arthrose nach Osteosynthese von supra-/intercondylären

Femurfrakturen (M.K. Zehntner, D. Marches, H.B. Burch und R. Ganz)

Spätergebnisse nach Osteosynthesen von distalen intraarticulären

Femurfrakturen (W. Mutschler, C. Burri, W. Birkner und J. Knötzele) 
Die monocondyläre Femurfraktur - eine Problemfraktur?

(J.R. Rether und P.J.Meeder)

Diskussion: Femur

Gefäßverletzungen

Operative Technik der Gefäßrekonstruktion und Frakturstabilisierung bei kniegelenksnahen Gefäßverletzungen (H.Zerkowski, J. Hanke,

N. Doetsch und R. Letsch)

Operative Taktik und Ergebnisse bei Gefäßverletzungen in Kombination

mit kniegelenksnahen Frakturen (G. Geiger, D. Silbernik und A. Hummel)

202

Komplexe Extremitätenverletzungen mit Gefäßläsionen im Kniebereich

(K. Kräch, U.von Wartburg, H. Eberle und V.Meyer)

A.poplitea Verletzungen bei distalen Femurfrakturen und Tibiakopffrakturen

(E. Scola, H.Zwipp, M. Holch und B. Wippermann)

Interdisziplinäres Management bei kniegelenksnahen Frakturen

mit Gefäßverletzungen (A. Bettermann und R. Moosdorf)

Sekundärprobleme

Kniegelenksnahe Frakturen, Korrekturoperationen nach fehlverheilten

Frakturen (G. Hierholzer und Ch. Chylarecki)

Therapeutisches Konzept bei posttraumatischen Gelenksteifen nach

kniegelenksnahen Frakturen (P.J. Meeder, S. Weller, H. Hermichen und K. Weise)

Therapeutisches Konzept bei der chronischen Bandinstabilität

nach kniegelenksnahen Frakturen (M. Börner und R.Ziegelmüller)

Die frühsekundäre operative Versorgung kniegelenksnaher Femurfrakturen

(A. Ekkernkamp, J. Brand, A. Lies und G. Muhr)

Die Hoffa-Fraktur - Unfallmechanik und Verletzungsmorphologie

(R. Fenkl, F. Baumgaertel und L. Gotzen)

Spätergebnisse zur Häufigkeit der posttraumatischen Arthrose nach

Tibiakopffrakturen (W. Knarse, F. Arman und Rahmanzadeh)

Komplikationen nach operativer Versorgung von Tibiakopffrakturen

(G. Ittner und R. Jasulka)

Operative Therapie der Kniegelenkskontrakturen nach Femurfrakturen (F. Vrevc)

Neue Gesichtspunkte zur Begutachtung der Spätfolgen

von kniegelenksnahen Frakturen (G. Hofmann und J. Probst)

Diskussion: Sekundärprobleme 
Die Indikation zur operativen Versorgung sportbedingter Rupturen des lateralen Bandhalteapparates am oberen Sprunggelenk

(B. Rosemeyer und W.Pförringer)

Sportbedingte osteochondrale Ausrisse des fibulotalaren Bandapparates bei Kindern (F. Gossé, C. Melzer und C.J. Wirth)

Die Bandplastik nach Watson-Jones für die laterale Instabilität am OSG beim Sportler (K. Weise, E. Lang und N. Karnatz)

5-Jahresergebnis nach wiederherstellender Bandchirurgie des fibularen Bandapparates am oberen Sprunggelenk bei Sportlern

(H. Thermann, H.Zwipp und H. Tscherne)

Diskussion: Oberes Sprunggelenk

Patella

Die Bedeutung des Patellastandes für die retropatellare Druckbelastung

(G.O. Hofmann)

Der retropatellare Knorpelschaden beim Sportler

(H. Cotta, J. Graf und E. Neusel)

Die traumatische Patellaluxation beim Sportler - eine Indikation zur Arthroskopie

(M. Neubert und K. Steinbrück)

Distalisierung und Medialisierung der Patella zur retropatellaren Druckentlastung (H.-J. Kock, K.M. Stürmer, J. Hanke, J. Letsch und R.S.Lorenz)

Luxation und Subluxation der Patella (W. Knopp, K. Neumann und G. Muhr) $\ldots .256$

Diskussion: Patella

Kniebänder

Der Stellenwert der Arthroskopie bei der frischen Kniegelenksinstabilität

(P. Münst, F. Bonnaire, A. Stiebitz und E.H. Kuner)

Indikationsstellung bei Sportverletzungen III - Knie-Bänder.

Diagnostisches Screening komplexer Knie-Band-Verletzungen

(P. Hochstein, H. Winkler und D. Jentschura)

Indikation und Technik der arthroskopischen Operation sportspezifischer Knieverletzungen unter besonderer Berücksichtigung der Laser-Chirurgie im Kniegelenk (H. Rudolph und H.J. Heberhold) 
Die partielle vordere Kreuzbandruptur nach Sportverletzung:

Differentialdiagnose - Therapie - Prognose (E. Lais, C. Petersen und P. Hertel) . . 268

Erweiterte Indikation zum vorderen Kreuzbandersatz durch verbesserte

Op-Technik und Rehabilitation (P.Lobenhoffer, N. Haas und H. Tscherne)

Sportfähigkeit nach vorderer Kreuzbandrekonstruktion

(K.P. Benedetto, W. Glötzer und Ch. Rangger)

Die isolierte vordere Kreuzbandruptur als Operationsindikation

nach Sportverletzungen (K.-K. Dittel)

Fünf Jahre Erfahrungen vorderer Kreuzbandrekonstruktion mit alloplastisch

verstärktem Sehnentransplantat (LAD) (K.-A. Riel, W. Hawe und P. Bernett)

Diskussion: Kniebänder

Obere Extremität

Sportfähigkeit nach Behandlung der Tossy-Verletzung mit resorbierbarem

Fixationsmaterial (M. Sangmeister, K. Hette, H. Knaepler und L. Gotzen)

Spätergebnisse nach operativer und konservativer Behandlung von

Sportverletzungen der Fingergelenke (H. Towfigh und K.-P. Schmit-Neuerburg) ...

Epidemiologie und Indikationsstellung bei Sportverletzungen der Hand und

des Ellenbogens (Th. Kreusser, E. Euler, K. Kress, V.B. Terzi und K. Wilhelm) ...

Überlastungsschäden im Bereich der Hand- und Fingergelenke beim Sportklettern;

klinische und radiologische Diagnostik (St. König und R. Schabus)

284

Indikation zur operativen Therapie beim Skidaumen

(Th. Reck, N.Pekel, B. Landsleitner und P.Schaller)

Diskussion: Obere Extremität

Überlastungsschäden

Überlastungsbedingte Schmerzsyndrome des Sportlers

(F.U. Niethard und A. Güßbacher)

Das chronische Kompartmentsyndrom beim Sportler

(W.Puhl, G. Wölffel und H.-P. Scharf)

Die Indikation zur operativen Therapie bei Insertionstendopathien

des Leistungssportlers (M. Kunz und H. Hess)

Diagnostik, Therapie, Ergebnisse der Epicondylitis humeri medialis

(Golferellenbogen) (K. Wilhelm)

Achillessehnenruptur bei Sportlem - Behandlung und Nachbehandlung

(A. Leitner, Ch. Voigt und A. Meißner) 
Der Achillessehnenriß als Sportverletzung - operative Behandlungstaktik

(J. Obrist, F. Genelin, A. Kröpfl und J.Zirknitzer)

Indikation und operative Möglichkeiten zur Rekonstruktion

veralteter Achillessehnen-Rupturen (J. Ahlers und G. Ritter)

Schultergelenk

Wertigkeit der klinischen und apparativen Untersuchungsmethoden beim Schulterschmerz des Sportlers (H. Resch, G. Sperner, K. Golser,

A. Oberhauser und K. Wicke)

Möglichkeiten der arthroskopischen Operation am Schultergelenk (W. Glinz)

Die Rotatorenmanschettenläsion im Sport - Indikation und differenziertes

therapeutisches Vorgehen (H.J. Refior)

Die Rockwood-Kapselplastik zur Rekonstruktion der multidirektionalen

Schulterinstabilität beim Leistungssportler (R. Kujat)

Ist die Operation nach Du Toit bei der Behandlung der posttraumatischrezidivierenden vorderen Schulterluxation des Sportlers indiziert?

(R. Theermann, H.J. Refior und A. Krödel)

Indikation zur Rekonstruktion bei sportspezifischen, kompletten und inkompletten Läsionen der Schulterrotatorenmanschette (Ch. Metzler und N. Wülker)

Diskussion: Schultergelenk

IV. Komplexe Handverletzungen

Akutversorgung

Osteosyntheseverfahren zur Stabilisierung bei komplexen Handverletzungen

(J.Rudigier)

Das Verletzungsmuster bei Explosionsverletzungen der Hand

(M. Geisenhauser, M. Schwarz und K. Lowka)

Hochdruckinjektionsverletzung der Hand, eine leicht unterschätzte Gefahr

(J. Ulmer und D. Buck-Gramcko)

Flußsäureverätzung der Hand (H.E. Mentzel, H.J. Backhaus und M. Trauner) . . .

Ergebnisse kombinierter Osteosyntheseverfahren zur Behandlung

komplexer Verletzungen an der Hand (S. Senst und H. Siebert)

Die Anwendung des Fixateur externe bei schweren Handverletzungen

(J.Grünert, W. Klein, D. Pennig und E. Brug)

Diskussion: Akutversorgung 
Grundsätze der Rekonstruktion komplexer Handverletzungen (U.Lanz)

Nachbehandlung von multistrukturellen Verletzungen der Hand (B.PetraciC) $\quad \ldots$.

Klassische und moderne Methoden des Gewebstransfers bei schweren

Handverletzungen (P. Flory, A. Berger und W. Schneider)

Funktionsgerechte Rekonstruktion komplexer Handverletzungen

(G. Germann, A. Schmidt-Barbo und G.Spilker)

Management der Versorgung einer schweren Handverletzung von der

Primärversorgung bis zur Rehabilitation in einer Klinik der AUVA

(W. Hintringer und M.Leixnering)

Die sekundäre Daumenverlängerung als Alternative zur Replantation

im Endgelenkbereich (K. Hette, Th. Lemke, H. Knaepler und L. Gotzen)

Die Adduktionskontraktur des Daumenstrahls nach komplexen Handverletzungen:

Wiederherstellungsmöglichkeiten mit ortsnahen Schwenklappen

(B. Helbig und R.T. Müller)

Primäre und sekundäre Möglichkeiten der Daumenersatzoperation nach Amputationsverletzungen (S. Eren und O. Paar)

V. Osteosynthese kindlicher Schaftfrakturen

Oberschenkel

Indikation, Zeitpunkt und Verfahrenswahl der Osteosynthese kindlicher

Femurschaftfrakturen (D. Havemann, M. Schmidt und W. Zenker)

Technik und Ergebnisse der Plattenosteosynthese am kindlichen Femur

(E.H. Kuner, H.-P. Mayer und W. Schlickewei)

Womit ist die Osteosynthese isolierter kindlicher Femurschaftfrakturen

zu rechtfertigen? (K. Tittel und F. Schauwecker)

Osteosynthese bei kindlichen Oberschenkelschaftfrakturen -

Konkurrenz zur konservativen Behandlung oder Verfahren der Wahl?

(H.D.Rahn, M. Kilic, G. Tolksdorff und F. Schauwecker)

Die Behandlung der kindlichen Oberschenkelfraktur mit dem

lateralen Klammerfixateur (Technik, Nachsorge, Ergebnisse)

(R. Neugebauer, U. Becker und A.Stinner)

Die Behandlung kindlicher Femurfrakturen mit der dynamisch axialen externen Fixation (W. Klein, D.Pennig, D. Baranowski und E. Brug)

Die intramedulläre Stabilisierung kindlicher Oberschenkelfrakturen mit der Bündelnagelung (W. Link, R. Schück und R. Wölfel) 
Spätergebnisse nach operativ versorgten kindlichen Oberschenkelschaftfrakturen

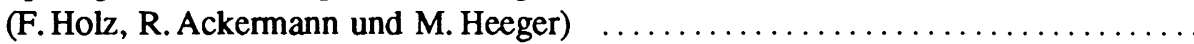

Über die Behandlung der kindlichen Oberschenkelschaftfrakturen

(L. Wessel und G. Scheuba)

Grenzen der spontanen Korrekturfähigkeit nach kindlichen Schaftfrakturen

der unteren Extremitäten - therapeutische Konsequenzen (L. von Laer)

Zur Operationsindikation bei der subtrochanteren Femurfraktur des Kindes

(N. Schwarz)

Spätergebnisse nach konservativer und operativer Therapie

kindlicher Femurschaftfrakturen (W.Zenker, Th. Buchhammer und Th. Gottorf) _.

Vergleich des Längenwachstums bei operativ und konservativ behandelten

Frakturen kindlicher Röhrenknochen (H. Resch, G. Spemer und K. Golser)

Ergebnisse operativer Behandlung von Schaftverletzungen der unteren Extremität

(K. Kunze, H.-J. Patzck, K. Schnccker und A. Bcttcrmann)

Korrektur bei Beinachsenfehlern im Kindesalter - Indikation und Technik

(R. Fuhrmann, F. Chicote-Campos und R. Venbrocks)

Diskussion: Posttraumatisches Fehlwachstum

Obere Extremität

Die operative Behandlung von Schaftfrakturen der oberen Extremität

im Kindesalter - Indikation, Zeitpunkt und Verfahrenswahl

(J. Eitenmüller, A. David, A. Sott und G. Muhr)

Technik und Ergebnisse der Osteosynthesen kindlicher Schaftfrakturen

an der oberen Extremität (V.Hendrich)

Alternativen der Versorgung kindlicher Oberarmschaftfrakturen

(R. Carbon, W. Link, P. Schaller und H. Beck)

Die Indikation zur Osteosynthese kindlicher Unterarmschaftfrakturen

(Th. Gottorf, W.Zenker, M. Schmidt und A. Peters)

Nachuntersuchungsergebnisse von 70 operativ stabilisierten kindlichen

Unterarmschaftfrakturen (M.Leixnering und N. Schwarz)

Die kindliche Unterarmschaftfraktur - Entscheidungsrichtlinien zur konservativen und operativen Therapie (G. Riedel, D. Birnbaum, E. Ludolph und G. Hierholzer) ..

Korrektur von Fehlstellungen nach kindlichen Schaftfrakturen der oberen

Extremität (H. Mittelmeier, E. Schmitt, J. Heisel und W. Mittelmeier) . .........

Diskussion: Obere Extremität 
Indikation, Zeitpunkt und Verfahrenswahl der Osteosynthese

kindlicher Tibiaschaftfrakturen (A. Rüter)

Technik und Ergebnisse der Osteosynthese kindlicher Schaftfrakturen

an der Tibia (R. Rahmanzadeh)

Versorgung kindlicher Unterschenkelschaftfrakturen mit schwerem

Weichteilschaden (A. David, J. Eitenmüller, H. BreitfuB und G. Muhr)

Die Therapie kindlicher Schaftfrakturen der unteren Extremitäten

mit Fixateur externe - auch bei unkomplizierten Frakturen?

(M. Echterhoff, H. Prinz und D. Jung)

Erfahrungen mit der Osteosynthese von 47 kindlichen Schaftfrakturen

an der unteren Extremität (P.M. Rommens, N. Van Leeuwen, P. Grymonprez

und P.L. Broos)

Nachuntersuchungsergebnisse und Verlaufsbeobachtungen bei kindlichen

Frakturen der proximalen Tibiametaphyse (M. Schmidt, L. von Laer,

D. Havemann und A. Peters)

Diskussion: Unterschenkel

VI. Experimentelle Unfallchirurgie

Neue Techniken

Subchondrale Durchblutung und posttraumatische Arthrose eine tierexperimentelle Untersuchung

(U. Freese, J. Graf, E. Neusel und F.U. Niethard)

Rasterelektronenmikroskopische Untersuchungen zum Einsatz verschiedener Lasersysteme bei der arthroskopischen Knorpelbearbeitung

(W.E. Siebert, J. Klanke, C. Scholz, D. Kohn, C.J. Wirth und G. Müller)

Ergebnisse des experimentellen Einsatzes eines Excimer-Lasers zur Knorpelabtragung (G. Hohlbach, K.O. Möller, G. Baretton und U. Schramm) ..

Die knöcherne Heilung nach Erbium: YAG-Laser Osteotomie am Kaninchen-Radius im Vergleich mit $\mathrm{CO}_{2}$-Laser-Systemen

(F. Dinkelaker, D.-R. Meyer, M. Grothues-Spork und C. Scholz)

Neue Erkenntnisse zur Calcaneusfraktur durch den CT-Daten-gesteuerten

Modellbau (W.Zenker, D. Bielstein und D. Havemann) .......................

Experimentelle Untersuchungen zur intraarticulären Fersenbeinfraktur (N. Wülker und H.Zwipp) 
Die Rolle der Fibula bei axialer Belastung des Unterschenkels, ermittelt aus der Korrelation der digitalisierten Druckverteilung im oberen Sprunggelenk (K.H. Widmer, V.Hendrich und H. Eisele)

Druck- und Kontaktflächenmessungen im oberen Sprunggelenk unter schrittweiser Durchtrennung der fibularen Bänder (R. Kasperk und O. Paar)

Das Kompartmentsyndrom - der Versuch einer non-invasiven Meßmethode des Gewebedruckes mittels mechanischer Impedanz (S. Winckler, U. Reder, S. Selter und G. Ruland)

Die lokale zellmediierte Immunität des osteomyelitischen Knochens Eine quantitative immunhistologische Analyse

(Ch. Josten, G. Muhr und Th. Griga)

Nuklearmedizinische Infektionsdiagnostik in der Unfallchirurgie. Szintigrafie mit Ceretec, Scintimun und Nanocoll (K.H. Winker, R. Reuland und S. Weller) 430

Diskussion: Neue Techniken

Neue Implantate

Untersuchung zum Knochen-Hydroxyl-Metallverbund. Tierexperimentelle

Untersuchung in der distalen Kaninchenfemurepiphyse (C. Voigt, W. Knarse, C. Müller-Mai und U. Gross)

Vergleichsuntersuchungen bei Osteosynthesen mittels Carbonfiber und Metallplatten an Kaninchen (J. Hankiss, I. Kadas, S. Frenyö, P. Fröhlich und J. Hamar)

Knochensubstanzverlust unter Plattenosteosynthese (F. Eitel, B. Steiner,

C. Wieland, L. Schweiberer, S. Peterhofen, L. Brunnberg, U. Matis und O. Pohler)

Ein extrem leichter und raumsparender Minifixateur externe mit nur einem Funktionselement (G. Schmidt)

Der EinfluB verschiedener Schraubentypen auf die Stabilität der

Fixateur-exteme-Osteosynthese und die Beanspruchung der Knochen

(L. Claes, H. Gerngroß und H. Kiefer)

Festigkeitsprüfung von drei verschiedenen Spongiosaschrauben zur Versorgung von Schenkelhalsbrüchen unter Berücksichtigung einer kanülierten selbstschneidenden Titanschraube (M.Leixnering, A. Schultz, L.W. Hamid und J. Poigenfürst)

Osteosynthese von Patella-Osteotomien mit resorbierbaren Materialien Ergebnisse einer tierexperimentellen Untersuchung (H.-U.Zieren, W. Holzmüller,

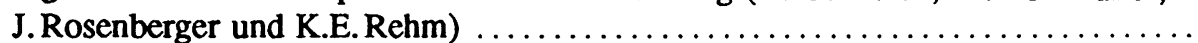

Die Versorgung von Sprunggelenksfrakturen unter Verwendung von Platten und Schrauben aus resorbierbarem Polymer-Material (J. Eitenmüller, A. David, A. Pommer und G. Muhr) 
Gewebekultur als Methode der Konservierung osteochondralen Gewebes Experimentelle Untersuchungen in vitro und in vivo

(J. Träger, R. Ascherl, K. Geißdörfer, G. Blümel und E. Hipp)

Extracorporale Bestrahlung von Knochengewebe - Experimentelle Untersuchungen an der Ratte (G. Voggenreiter, R. Ascherl, K. Geißdörfer und M.A. Scherer) . . ... 445

Konfektionierte Spongiosaplastik (R. Giers und V.Echtermeyer)

Immunisierung gegen Blutgruppenantigene durch allogene

Knochentransplantation (H. Knaepler, R. Ascherl, V. Kretschmer und L. Gotzen) . .

Temporäre und definitive Deckung verbrannter Körperoberfläche durch allogene und alloplastische Materialien - Experimentelle Studie an der Ratte

(J.W. Weidringer, R. Ascherl, A. Stemberger, G. Blümel und A. Eder)

Druckläsionen des $\mathrm{N}$. ischiadicus des Haushuhns - elektromyographische und morphologische Untersuchungen (H.-E. Nau, F. Rauhut, M. Konerding und M. Blank)

Freic Nerventransplantation nach Kältekonservierung

(V.Kobor, R. Ascherl, U. Reichenauer und G. Blümel)

Homologe knochengestielte vordere Kreuzbandtransplantation

am Kaninchenmodell (B. Fromm, B. Krause und W. Kummer)

Patellarsehnenverkürzungen nach Transplantatentnahme

(K.E. Rehm und W. Holzmüller)

Vergleichende, altersabhängige biomechanische Belastungsuntersuchung der Kreuzbänder und der Patellarsehnen (W.J. Kasperczyk, S. Rosocha,

L. Borchers, U. Bosch und H.J. Oestern)

Selbstarretierende Fixationsklammern für Bänder und Kunstbänder im Vergleich (D. Hempel und C. Mattheck)

Die Genauigkeit der nicht instrumentierten Innen-Außenbohrung bei der Positionierung des proximalen vorderen Kreuzbandansatzes (M. Bernard, P. Hertel und E. Lais)

Biomechanische Eigenschaften des vorderen Kreuzbandes in Abhängigkeit von der Winkelstellung des Tibia-Femur-Systems und der Belastungsrichtung (H.J.Früh, W. Siebels, J.Franke, R. Ascherl und G. Blümel)

Diskussion: Gewebeersatz

Computergestützte dreidimensionale Geometrieanalyse von Oberschenkelknochen zur Gestaltung einer zementfreien Hüftendoprothese (T. Gerhard, W. Siebels, G. Hemdl und R. Ascherl) 
Entwurf und Berechnung einer neuen flexiblen Scnaftprothese

für das Hüftgelenk (R. Thull und G.Zeller)

Bedeutung des Trochanter-Zuges auf den KraftfluB im proximalen Femur nach

Prothesenimplantation (A. Bettermann, H. Ecke, A.Sablozki und M. Nietert)

Die zementfrei implantierbare bitrochantere Hüftendoprothese -

Erfahrungen und Ergebnisse seit 1982 (H. Ecke und B. Rieck)

Reduktion der Ossifikationen nach zementfreiem Hüftgelenkersatz

mittels Fibrinkleber (J. Rödig, F. Dinkelaker und R. Rahmanzadeh)

Immunhistochemische Untersuchungen zur Frage der Abstoßungsreaktionen

bei der Lockerung von Gelenkendoprothesen (F. Löer, K.M. Peters, F. Hofstädter

und E. Savvidis)

Biomechanische Betrachtungen zur Druckeinleitung und Druckerhaltung

bei intramedullären Kompressionsosteosynthesen (G. Ritter)

Vergleichende biomechanische Messungen der Torsionsstabilität

von intramedullären Nagel-Osteosynthesen (H. Mittelmeier, M. Trennheuser

und W. Mittelmeier)

Perioperative und intraoperative kontinuierliche Gewebedruckmessung

bei der gedeckten Tibiamarknagelung (F. Bonnaire, E.H. Kuner und P.Münst)

Schock

Zur Pathogenese von Reperfusionsschäden nach partieller Leberischämie

mit intermem Shunt (H.P. Friedl, O. Trentz, L.H. Toledo-Pereyra und G.O. Till)

Experimentelle Untersuchungen zu Auswirkungen der hohen Aortenblockade im schweren hämorrhagischen Schock (I. Marzi, V. Bühren, F. Blessing,

O. Gonschorek und O. Trentz)

Muster und Funktion alveolärer Phagocyten nach multiplem Trauma

(A. Dwenger, C. Beychok, A. Vorbeck und G. Regel)

Komplementsystem, Histamin und Xanthinoxidase als initiale

Trigger-Mechanismen des akuten Lungenversagens

(H.P. Friedl, G.O. Till, O. Trentz und P.A. Ward)

Veränderungen der Lymphocytensubpopulationen beim Polytrauma

(M. Cebulla, L. Bergmann, K. Fredeking, P. Konold und A. Pannike)

Diskussion: Schock

Die Wertigkeit verschiedener Versuchstierspecies für experimentelle

Untersuchungen am Knochen (H.Wissing, K.M. Stürmer und G. Breidenstein) 
Intravitale Bewegungsmessung bei der Frakturheilung

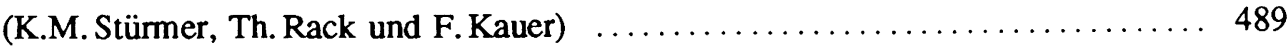

Mechanische In-vitro-Meßverfahren am Knochen (L.Claes) $\ldots \ldots \ldots \ldots \ldots \ldots .498$

Diskussion: Grundlagen experimenteller Methodik am Knochen ............. 499

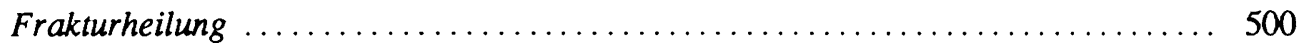

Frakturheilung bei progressiv dynamisierter Schienung (P. Fröhlich) $\ldots \ldots \ldots \ldots 500$

Knochenbruchheilung und Elektrostimulation - Experimentelle Untersuchungen

(M. Friedrich, R. Ascherl, D. Sowa und W. Siebels) _................. 501

Schnelleres Längenwachstum durch Elektrostimulation?

Tierexperimentelle Studie (D. Sowa, R. Ascherl, M. Friedrich und G. Blümel) $\ldots . \quad 502$

Ist die „wasserdichte" Reposition von Epiphysenfugenverletzungen

zwingende Voraussetzung für einen störungsfreien Heilverlauf?

(M. Dallek, C. Pallacks und K.H. Jungbluth) $\ldots \ldots \ldots \ldots \ldots \ldots \ldots \ldots \ldots \ldots$

Die Sonographie beim kindlichen Oberschenkelbruch

(B.W. Wippermann, R. Hoffmann, P. Reimer und N. Haas) _............. 504

Der Stellenwert der Corticotomie und Osteotomie nach Segmentverschiebung bei der Behandlung von Knoch:endefekten (R. Brutscher, A. Rüter und S.M.Perren) _. 505

Segmentverschiebung (Ilizarov) bei großen Schaftdefekten langer Röhrenknochen: Knochenregeneration auch am Marknagel (U. Brunner, S. KeBler, L. Schweiberer,

B. Rahn und S.M.Perren)

The Partridge Osteosynthesis. An Experimental Study on Vascularization and Biomechanics of Cerclage Wire and Bands in Animals, and a Clinical Study in 66 Patients. The Solution for Subprosthetic Femur Fractures?

(V.A. de Ridder, P.J.Klopper, F.W. Heatley and A.R. Koomen)

Röntgenmorphologische, mikroangiographische und histomorphologische

Untersuchungen der Anbauvorgänge nach unphysiologischer Belastung der Ulna

(R. Seibold und F. Eitel)

Quantitative Fluorescenzbildanalyse an Knochenschnitten

(K. Wolf, M. Saleh, W. Stock, R. Hierner, B. Breuckmann und L. Schweiberer) ....

Regeneration des Knochens nach thermischer Schädigung. Eine tierexperimentelle Untersuchung (R. Büttenmeyer, J. Hendricks, J.C. Bruck und F. Weyer)

\section{Vorlesungen}

The Adult Respiratory Distress and Multiple Organ Failure Syndrome (R.J.A. Goris) 
In-vivo-Messungen an Implantaten zur Rekonstruktion von

Wirbelsäulenfrakturen (H.-J. Wilke, L. Claes und O. Wörsdörfer)

Cyclische Belastungstests verschiedener Wirbelsäulenimplantate -

Fatigue Strength of Various Spinal Implants

(R.H. Wittenberg, M.S. Coffee, W.T.Edwards und A.A. White)

Ventrale transpediculäre Stabilisation im Bereich der LWS

am anatomischen Präparat (H. Hertlein, G. Lob und F. Zogelmeier)

Die Discusverletzung als wesentliche Traumfolge bei thorakalen und lumbalen

Wirbelfrakturen (S. v.Gumppenberg, B. Allgayer, E.v.d. Fliert und B.Claudi)

24stündige Antibioticaprophylaxe bei der operativen Versorgung

proximaler Femurfrakturen (M. Heberer, A. Bodoky, U.Neff und F. Harder)

Analyse der „Staphylokokken-Schutzhülle“ bei Kulturen auf medizinischen

Kunststoffimplantaten (J. Sauer, R. Inglis, P. Konold, A. Pannike, I. Löw,

V.Schäfer und G.Zimmermann)

Indikationen und Ergebnisse der Baclofen-Pumpen-Implantation zur Therapie der Spastik bei Rückenmarkverletzungen (St. Elenz, H.J. Böhm und G. Hierholzer)

Diskussion: Freie Themen

\section{Kuratorium ZNS: Notwendigkeiten und Möglichkeiten}

der Frührehabilitation

Notwendigkeiten und Möglichkeiten der Frührehabilitation

schwer Schädelhirnverletzter (K. Mayer)

Frührehabilitation für schwer Schädel-Hirn-Verletzte in der Unfallklinik

(A. Wentzensen)

Frührehabilitation bei Schädelhirntrauma - aus neurochirurgischer Sicht

(M.R. Gaab)

Frührehabilitation für schwer schädelhirnverletzte Patienten

in der Nachsorgeklinik (W. Gobiet)

Diskussion: Kuratorium ZNS

EDV in der Unfallchirurgie - Anwendungsmöglichkeiten und Anforderungen

(R. Schunck und R. Haunhorst)

EDV in der Unfallchirurgie - gegenwärtig verwirklichte Lösungen

(P.-M. Hax, G. Hierholzer und A. Woytewicz) 
Entwicklung und Anwendung eines hierarchischen Codierungsschlüssels zur EDV-Dokumentation in der Unfallchirurgie

(R. Schlenzka, M. Poll, M. Schnabel und L. Gotzen)

Ärztliche Anforderungen an eine Dokumentation in der Unfallchirurgie

(N.M.Meenen und J. Berger)

EDV in der Medizin, Fehlerreduzierung durch automatische Datenerfassung

am Patienten (R. Inglis, M.Pannike, J. Windolf und A. Pannike)

Medizinische Dokumentation mit dem H.A.N.D.-System - Nutzen, Erfahrung in

Routine und Wissenschaft (M.V.Knopp, S.Potzer, M. Martens und H. Frobenius)

Rationelles Expertensystem für die traumatologische Dokumentation

(E. Remmel und H.-W. Stedtfeld)

EDV-unterstützte Erfassung und Analyse postoperativer Komplikationen

in der Unfallchirurgie (Ch. Jürgens, H.-R. Kortmann, D. Wolter

und A. Biewener)

Diskussion: EDV-Dokumentationssysteme in der Unfallchirurgie I

Spezielle Anwendungen und Demonstrationen

Dokumentation der AO - derzeitiger Stand und Weiterentwicklung

(P. Matter und M. Bühler)

Anwenderorientierte Datenverarbeitung zur Klinikorganisation -

eine interaktive Demonstration (N.M. Meenen, M. Faltis und K.H. Jungbluth)

Diskussion: Spezielle Anwendungen und Demonstrationen

Makroreplantation der oberen Extemität (A. Ahmadi)

Kritische Analyse der Spätergebnisse nach Replantation (J.Probst)

Zur Indikation verschiedener Osteosyntheseverfahren bei Fingerreplantationen

(J. Degreif, J. Rudigier und V. Karnosky)

Beitrag zur Wertigkeit von Replantationen bei isolierten Langfingeramputationen

(F. Genelin, F. Gasperschitz und R. Helmberger)

Komplexe Handverletzungen, Replantation, mikrochirurgische Revascularisation, funktionelle Ergebnisse (Th. Kreusser, W. Stock, M. Legner und P. Thaller) .....

Diskussion: Replantation heute: Indikation, Technik, Ergebnisse 
Anforderungen an präklinische Versorgung und Transport Schwerverletzter

(U. Obertacke und K.-P.Schmit-Neuerburg)

Präklinisches Scoring - Aufgabe des Notarztes

(B. Bouillon, M. Schweins, A. Lechleuthner und H. Troidl)

Polytrauma-Scores - Übersicht und Versuch der Abschätzung ihrer Wertigkeit

(M.A. Scherer, R. Ascherl, M. Leonard und G. Blümel

Early Mortality after Polytrauma: A Retrospective Study

(P.M.Rommens, M.J. Miserez, H.H.Delooz und P.I. Broos)

Notfallmedizinische Versorgung des Schwerstverletzten in einem ländlich strukturierten Gebiet. Hat die Einführung des NEF-Systems die präklinische Versorgung verbessert? (U. Malewski, K. Hette, M. Sangmeister und L. Gotzen)

Die Wertigkeit des notärztlichen neurologischen Erstbefundes beim schädel-hirnverletzten Patienten für die Prioritätenfestlegung bei der Aufnahme in der Klinik (W.D. Hirsch und H. Bauer)

Wahl der Zielklinik - Aufgabe des Notarztes

(M. Schweins, B. Bouillon, A. Lechleuthner und Th. Tiling)

Bedarfsgerechte Versorgung von Katastrophenopfern - Klinikmanagement am Beispiel des Flugschauunfalles von Ramstein (V. Bühren, T. Koßmann, I. Wittling und O. Trentz)

Aus Fehlern lernen. 10-Jahres-Analyse der präklinischen Erstversorgung

Polytraumatisierter einer westdeutschen Großstadt (R.H.Gahr)

Diskussion: Präklinische Versorgung Schwerstverletzter

Hat die Unfallchirurgie eine Zukunft?

Stellungnahme aus der Sicht des klinisch tätigen Orthopäden (H. Cotta)

Stellungnahme aus der Sicht des Unfallchirurgen

einer Berufsgenossenschaftlichen Klinik (G. Hierholzer)

Stellungnahme aus der Sicht des Universitäts-Unfallchirurgen

(H. Tscherne und A. Tempka)

Stellungnahme aus der Sicht des Universitätschirurgen (L. Schweiberer)

Stellungnahme aus der Sicht des Chefarztes für Chirurgie

am regionalen Krankenhaus ( $R$.Zwirner)

Stellungnahme aus der Sicht des Oberarztes an einer Unfallchirurgischen Universitätsklinik (K.M.Stürmer) 
Kriterien für die Begutachtung und Richtwerte für Invalidität und

Erwerbsminderung durch Funktionsverlust nach schwerer Handverletzung

(H.G. Haas und K.H.Lennert)

Funktionsverbessernde Operationen nach schweren Handverletzungen:

Zumutbarkeit und Auswirkungen auf die Verletztenrente (J.Geldmacher)

Die MdE bei Vorschaden (Ch. Wulle)

Eine neue Bewertungsskala zur Begutachtung der brandverletzten Hand

(A. Grabosch und J.C. Bruck)

Einfache und schnelle Methode zur Objektivierung von Sensibilitätsstörungen

(A. Olinger, C. Braun, W. Mittelmaier und V. Bühren)

Die Weiterentwicklung der Berufskrankheiten

BK Nr. 2102: Versicherte Tätigkeit - versicherter Schaden.

Die Frage nach der Kausalität (E. Ludolph)

Die Kniegelenkarthrose als Berufskrankheit? (H. Greinemann)

Die Bedeutung der HIV-Infektion als Berufskrankheit des Krankenhauspersonals (J. Windolf, R. Inglis, J.M. Rueger und A. Pannike)

Vasculärer Spätschaden nach traumatischer Beinamputation

(E. Paes, W. Mutschler, J.F. Vollmar und P. Pauschinger)

Die aseptischen Knorpelknochennekrosen und die Arbeitsmedizin (H.Schiller) _. 653

Diskussion: Die Weiterentwicklung der Berufskrankheiten

Forensische Bedeutung der nicht beachteten tiefen Beinvenenthrombose

bei ambulanten Patienten (G. Carstensen)

Thromboseprophylaxe bei ambulanten Patienten. Thrombosehäufigkeit,

Risikofaktoren, klinische und apparative Diagnostik (H.-G. Breyer)

Thromboseprophylaxe bei ambulanten Patienten - Befürwortung

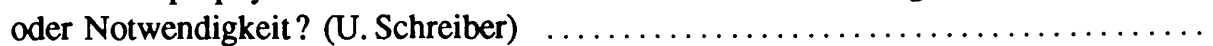

Thromboembolieprophylaxe bei ambulanten und poststationären Patienten.

Indikation, Art und Dauer der Prophylaxe (H. Reilmann und E. Förster)

Artzhaftpflicht und strafrechtliche Konsequenzen bei unterlassener

Thromboseprophylaxe (K. Ulsenheimer) 
Septische Prothesenlockerung - Anwendung von Kollagen-Gentamicin (R. Ascherl, A. Stemberger, M.A. Scherer und G. Blümel)

Thoracolumbale Wirbelsäulenverletzungen: Erstbehandlung und definitive Versorgung (E. Birk, R. Randt, E. Fecht und V. Echtermeyer)

Biodynamik der Druck- und Zugepiphyse am kindlichen proximalen Femur (M. Dallek und K.H. Jungbluth)

Zur Biomechanik autoklavierter, bestrahlter und kältekonservierter Corticalis

(H.J.Früh, G. Voggenreiter, R. Ascherl, M.A. Scherer und W. Siebels)

Transmissionselektronenmikroskopische Untersuchungen an kältekonservierten allogenen Nerventransplantaten (F. Hammersen, R. Ascherl, V. Kobor, M.A. Scherer und G. Blümel)

Arthroskopische Befunde bei Meniscustransplantation und Meniscusersatz (K.A. Milachowski, D. Kohn und C. J. Wirth)

Sportverletzungen - Praktikabilität, Möglichkeiten und Konsequenzen einer EDV-Erfassung (J.Pöhlmann, M. Schnabel, M. Ennis und L. Gotzen)

Ultraschall - Experimentelle und klinische Ergebnisse nach Spongiosatransplantation (H.B. Reith, W. Böddeker und W. Kozuschek) ....

Klinische und experimentelle Aspekte der Lungenkontusion

(M. Reuter, U. Obertacke und Th. Joka)

Zur Osteosynthese von Schenkelhalsfrakturen mit der dynamischen Hüftschraube Ergebnisse aus 6 Jahren (C.G. Schulze, G. Siebler und E.H. Kuner)

Die operative Behandlung einer typischen Sportverletzung, der kompletten akromioclavicularen Luxation - Indikation, Technik und Ergebnisse bei Anwendung der Balserplatte (M. Walz und M. Schax)

Die Talusfraktur - Funktionsanalyse nach Therapie mit Hilfe der dynamischen Pedographie (Th. Mittmeier, M. Fäßler und G. Lob)

Schlußveranstaltung

Schlußworte von Prof. Dr. med. A. Pannike

Bericht über die Mitgliederversammlung der Deutschen Gesellschaft

für Unfallheilkunde e.V. am 23.11.1989 in Berlin 


\title{
Mediatoren des traumatisch-hämorrhagischen Schockgeschehens mit neuer klinischer Relevanz
}

\author{
D. Nast-Kolb, Ch. Waydhas, M. Jochum, K.-H. Duswald und L. Schweiberer
}

Chirurgische Klinik und Poliklinik, Klinikum Innenstadt der Ludiwg-Maximilian-Universität, Nußbaumstraße 20, D-8000 München 2

In einer prospektiven Polytraumastudie wurden 69 Patienten (mittlerer ISS: 36 ) untersucht. Davon verstarben 11 zwischen dem 4. und 28. Tag im Multiorganversagen. 29 Verletzte überlebten definierte Organfunktionsstörungen, 29 Patienten hatten einen komplikationslosen Verlauf.

Die Mittelwertskurvenverläufe einer Vielzahl biochemischer Faktoren unterschieden signifikant zwischen den 3 Gruppen mit prognostischer Relevanz:

Bereits bei Klinikaufnahme ermöglichten die Proteinasen Kathepsin B (Sensistivität: $63 \%$, Pos. präd. Wert: $86 \%$ ) und PMN-Elastase (84\%/67\%), ebenso wie Mediatoren der

Tabelle 1

\begin{tabular}{lll}
\hline & Sensitivität & Pos. präd. Wert \\
\hline Laktat $\left(^{*}\right)$ & $60 \%$ & $60 \%$ \\
PMN-Elastase & $73 \%$ & $67 \%$ \\
C-reaktives Protein & $67 \%$ & $86 \%$ \\
Pancreatic Secretory & $67 \%$ & $67 \%$ \\
Trypsin Inhibitor & $75 \%$ & $67 \%$ \\
Neopterin & & \\
\hline
\end{tabular}


Gerinnung, AT III (71\%/71\%) und Prothrombin (71\%/73\%), sowie der Fibrinolyse, DDFragment $(62 \% / 70 \%)$ und t-PA (62\%/68\%), eine Vorhersage späteren Organversagens.

Bezüglich sekundärem Versterben ergaben sich bei Klinikaufnahme $\left({ }^{*}\right)$ bzw. am 3. Tag nach dem Trauma folgende prognostische Ergebnisse (Tabelle 1).

Durch die Kombination mehrerer Faktoren lieb sich die Vorhersage späteren Versterbens weiter verbessern: War kein Parameter pathologisch, so überlebten alle (Neg. präd. Wert: 100\%). Dagegen verstarben sämtliche Patienten bei mindestens 4 pathologischen Werten (Pos. präd. Wert: 100\%). 